\title{
An Experimental Investigation on Transpiration Cooling Part II: Comparison of Cooling Methods and Media
}

\author{
J. H. Wang \\ The 13th Department of University of Science and Technology of China, People's Republic of China
}

\author{
J. Messner and H. Stetter \\ Institut für Thermische Strömungsmaschinen und Maschinenlaboratorium, University of Stuttgart, \\ Stuttgart, Germany
}

This article attempts to provide a cooling performance comparison of various mass transfer cooling methods and different cooling media through two experiments. In the first experiment, pressurized air was used as a cooling medium and two different circular tubes were used as specimens. One is made of impermeable solid material with four rows of discrete holes to simulate film cooling, and the other consists of sintered porous material to create a porous transpiration cooling effect. The natures of transpiration cooling and film cooling including leading and trailing edge injection cooling were compared. This experiment found that by using a gaseous cooling medium, transpiration cooling could provide a higher cooling effect and a larger coolant coverage than film cooling in the leading stagnation region, and on the side of the specimen at the same coolant injection flow rates; but in the trailing stagnation region, the traditional coolant injection method through discrete film holes might be better than transpiration cooling, especially for turbine blades with thin trailing edges. In the second experiment, the cooling effects of gaseous and liquid media on the same porous tube's surface were compared. This experiment showed that the porous areas cooled using gaseous and liquid cooling media were almost identical, but the cooling effect of liquid evaporation was much higher than that of gaseous cooling, espe-

Received 25 June 2002; accepted 1 July 2002.

The authors acknowledge Dr. Mayer and Dr. Eyb for their suggestions and software engineers Mr. Rath and Mr. Findeisen for their assistance in this research work.

Address correspondence to J. Messner, Institut für Thermische Strömungsmaschinen und Maschinenlaboratorium, University of Stuttgart, Pfaffenwaldring 6, Stuttgart-Valhingen, D 70569, Germany. E-mail: messner@itsm.uni-stuttgart.de cially in the leading and trailing stagnation regions of turbine blades. This important discovery makes it possible to solve the stagnation region problems in turbine blade cooling.

Keywords Transpiration cooling, Film cooling, Liquid coolant, Gaseous coolant

Today, film cooling is one of the cooling schemes being widely used to protect turbine components from high turbine inlet temperature. The performance of film cooling is usually influenced by many factors, such as wall curvature, hole size, shape, location, injection angle, three-dimensional external flow structure, flow unsteadiness, turbulence, compressibility, and so on. It seems to be very difficult to control these factors and to obtain an ideal film cooling effect thereby. Thus, there have been investigations to find a more promising cooling method for years. Leontiev (1999) predicts that "as compared to other methods, transpiration cooling is the most effective cooling technique, and makes it possible to obtain required wall temperature with minimal discharge of coolant." This article presents a comparison of transpiration cooling and film cooling.

In modern gas turbines, the consumption of cooling media is very high. This high consumption has made cooling turbine components with compressor discharge air very challenging. In this situation, water was considered as a candidate to substitute for gaseous coolant in the protection of the nozzle guide vanes of industrial gas turbine engines. For example, in studies by Van Fossen (1983), Biesiadny et al. (1987), and Nirmalan et al. (1998), the cooling performance of a water-air mixture as cooling medium was investigated. It is clear that water was the most effective coolant because liquid evaporating can provide a very large heat sink. But in modern gas turbine, advanced materials, such as nickel super-alloys and various ceramics, have 
been widely employed. In general, the thermal conductivity and diffusivity of these impermeable materials are relatively low, thus water evaporation could lead to thermal failure of the turbine components due to the large temperature gradients brought by water droplets created during the water evaporation process. In comparison with these materials, sintered porous materials have much higher thermal conductivity and diffusivity, and the water droplet sizes can also be homogenized through the pores of the materials. These important characteristics provide the opportunity to use water directly as a cooling medium for the second transpiration cooling experiment.

In the first part of this article, an application of the infrared thermal imaging technique was presented. In this part, the performances of film cooling and transpiration cooling were quantitatively compared, and the difference between gaseous and liquid cooling media in porous transpiration cooling was experimentally analyzed using this measurement technique. The goal of this work is to provide a quantitative comparison for the researchers and designers of the turbine components with mass transfer cooling function to choose a cooling method and medium, to improve coolant chamber configuration design, and to control coolant consumption level.

\section{EXPERIMENTAL FACILITIES}

In the first experiment, two circular tube were used as specimens. One is made of sintered chromium-nickel steel with a porosity of $21 \%$ to create a porous transpiration cooling effect, and the other consists of solid material with four rows of discrete injection holes to simulate film cooling effect in different regions, namely in the leading and trailing stagnation regions, and on the side of gas turbine blades. The holes on the leading and trailing lines have two staggered inclined angles of $\pm 15^{\circ}$ in the mainstream direction, as shown in Figure 1a. The ellipse in the figure is an enlarged location of the front view from direction A. The two tubes have the same exterior size: an

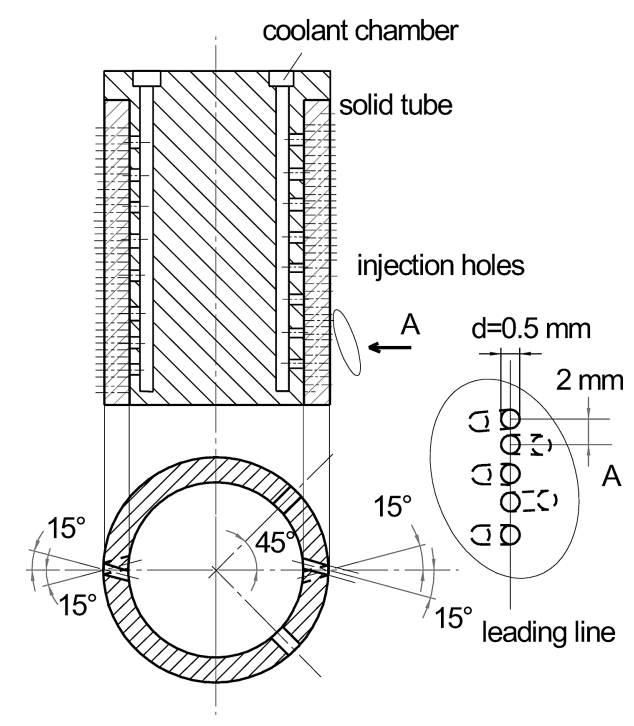

FIGURE 1a

Construction of solid tube.

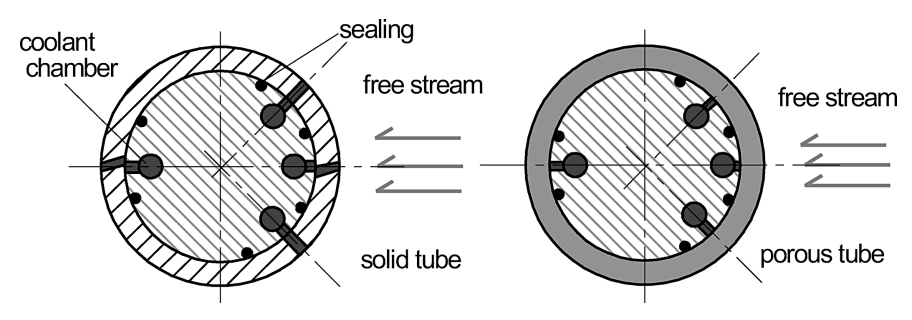

FIGURE 1b

Cross section of two specimens and coolant chamber.

outside diameter of $36.5 \mathrm{~mm}$, a height of $52 \mathrm{~mm}$, and a thickness of $3.5 \mathrm{~mm}$. Several closed sealings were used to control the injected coolant to flow into the desired region. The configurations of coolant chambers and sealings are shown in Figure $1 \mathrm{~b}$.

In the second experiment, gaseous and liquid cooling media were successively injected into the same porous specimen, and the cooling performances were compared. The air permeability characteristics of the porous material were shown in the first part of this article, and the water permeability characteristics are illustrated in Figure 1c. The experiments were carried out in the hot gas wind tunnel at the Institute of Thermal Turbomachinery at the University of Stuttgart. A schematic layout of the wind tunnel was described in the first part of this article.

\section{RESULTS AND DISCUSSIONS}

There are three IR transmissible windows in the test section of the wind tunnel. Through the three windows, the leading, side, and trailing regions of the specimen can be observed. The relative positions of the IR scanner and the specimen are shown in Figure 2. The influence of these measurement positions on measurement accuracy was analyzed, and the corresponding calibration methods were described in the first part of this article.

\section{Comparison of Cooling Methods}

The first experiment was conducted at a free stream temperature of $240^{\circ} \mathrm{C}$ with a mass flow rate of $400 \mathrm{~g} / \mathrm{s}$. Gaseous coolant was injected into the coolant chambers at a temperature of $30^{\circ} \mathrm{C}$. Surface temperatures were measured using the VARIOSCAN 3021 IR system. Original thermal images consist of $240 \times 360$ pixels. Only the pixels on the specimen surface were acquired and processed. The true orientations of the corresponding pixels and the areas counted in the average cooling

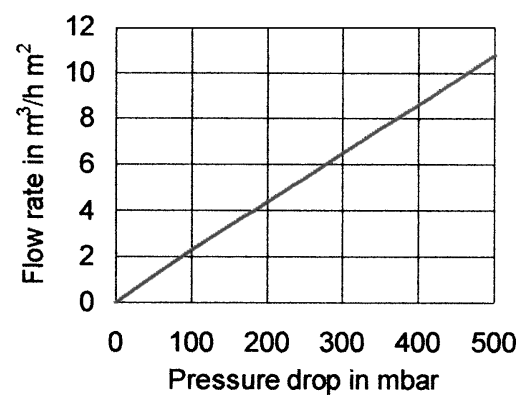

FIGURE 1c

Water permeability. 


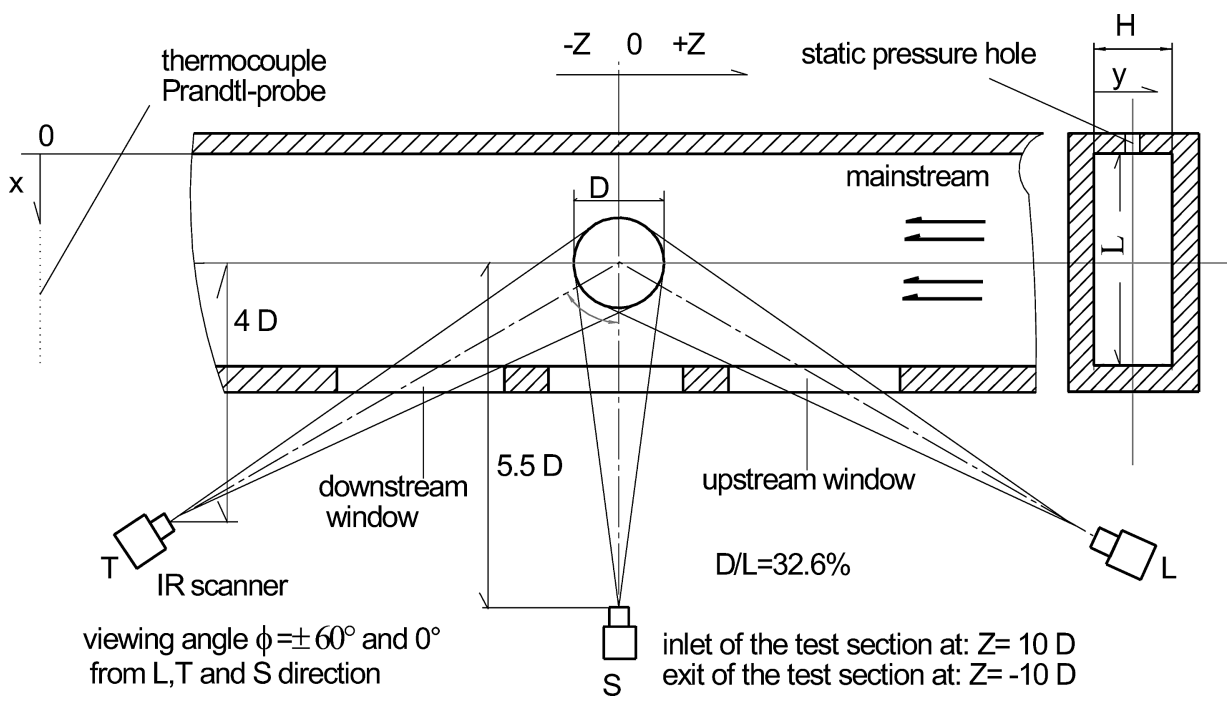

FIGURE 2

Measurement angles and positions.

effect were calculated using a simple triangular relationship. This relationship between the true orientations and the corresponding pixels are arranged in the appendix of this article. If the temperature difference between free stream and coolant could be considered constant, local cooling effect, as defined in the first part of this article as $\eta_{\text {loc }}=\left(\mathrm{T}_{\mathrm{iw}}-\mathrm{T}_{\mathrm{cw}}\right) /\left(\mathrm{T}_{\infty}-\mathrm{T}_{\mathrm{c}}\right)$, may be illustrated through a contour plot of the temperature drop from the initial wall to the cooled wall.

\section{Cooling Effect in Leading Region}

When a gaseous cooling medium was injected through the discrete film holes at the leading line of the solid tube, the corresponding cooling performance was similar to the leading injection cooling of gas turbine blades. Figure 3 shows two series of temperature drop contour plots at different coolant injection rates, $1.5 \mathrm{~g} / \mathrm{s}, 2.0 \mathrm{~g} / \mathrm{s}, 2.5 \mathrm{~g} / \mathrm{s}, 3.0 \mathrm{~g} / \mathrm{s}$, and $3.5 \mathrm{~g} / \mathrm{s}$, respectively. The upper series is from film cooling, and the lower is from

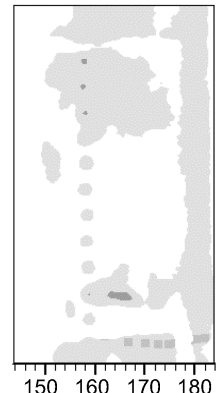

$\begin{array}{llll}150 & 160 & 170 & 180\end{array}$

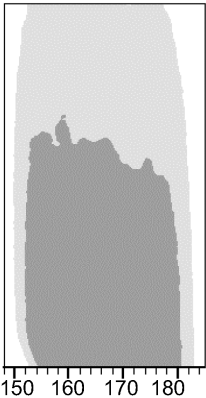

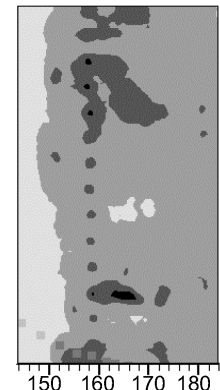
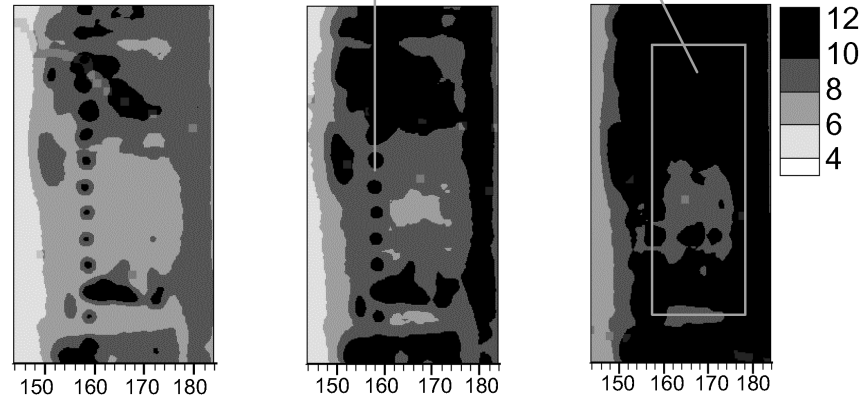

$\mathrm{T}_{\mathrm{in}}-\mathrm{T}_{\mathrm{cw}}$ in $\mathrm{K}$

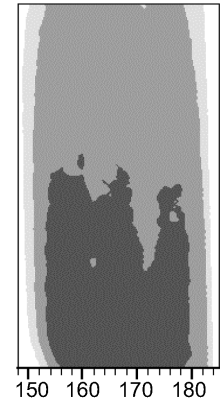

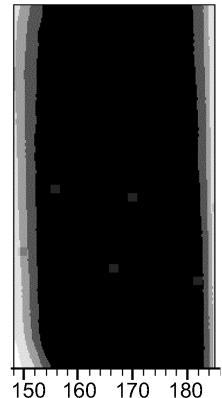
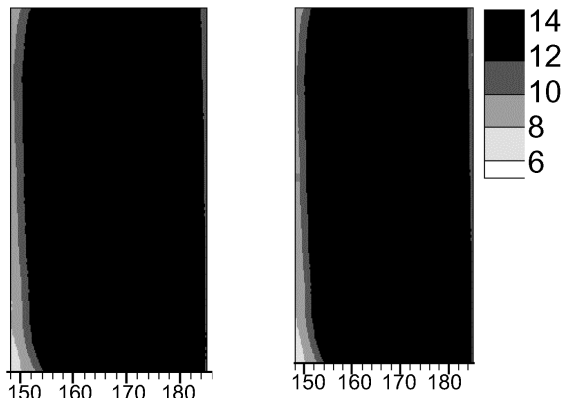

FIGURE 3

Local cooling effects in leading stagnation region; above: film cooling. 
TABLE 1

Averaging Effect in Leading Region

\begin{tabular}{llllll}
\hline & $1.5 \mathrm{~g} / \mathrm{s}$ & $2.0 \mathrm{~g} / \mathrm{s}$ & $2.5 \mathrm{~g} / \mathrm{s}$ & $3.0 \mathrm{~g} / \mathrm{s}$ & $3.5 \mathrm{~g} / \mathrm{s}$ \\
\hline FL & 0.0053 & 0.0154 & 0.0189 & 0.0237 & 0.0295 \\
TL & 0.0332 & 0.0423 & 0.0642 & 0.0809 & 0.0823 \\
\hline
\end{tabular}

transpiration cooling. Original IR images were captured from the upstream window of the specimens with a viewing angel of $60^{\circ}$, while only the coolant chambers at the leading lines were supplied with gaseous cooling medium. The pixels illustrated in the contour plots were acquired from the pixel number 70 to 150 in vertical direction, from 150 to 185 in horizontal direction from the original images, and the leading line stood on the pixel number 163.

It is clear that porous transpiration cooling could achieve a higher temperature drop, a larger protected area, and a more homogenous cooling effect in the leading stagnation region. This result could be quantitatively demonstrated with data from average cooling effect, as shown in Table 1 . This average effect was calculated according to the definition of $\eta_{\mathrm{avg}}=\int_{\mathrm{A}}\left(\mathrm{T}_{\mathrm{iw}}-\right.$ $\left.\mathrm{T}_{\mathrm{cw}}\right) \mathrm{dA} /\left(\mathrm{T}_{\infty}-\mathrm{T}_{\mathrm{c}}\right) \mathrm{A}$, and the areas counted in the cooling effect were calculated through a simple triangular relation.

Transpiration cooling obtained higher cooling effects at different coolant flow rates. The reason is that transpiration cooling effects could be maintained at the pores outlets of the porous wall, and the entire stagnation region was covered with the pores. In the case of the leading injection cooling on the solid tube, coolant penetrated the single row of film holes, mixed with the mainstream, and transported the heat on the surface through the flow of the mainstream. Because the mainstream velocities in this region were low to nil, thus it was difficult to remove a large amount of heat from the surface of the solid tube.

\section{Cooling Effect on the Side of the Specimen}

If the chambers at $\pm 45^{\circ}$ from the leading line of the two tubes were supplied with coolant, the corresponding effects may be seen on the curvatures of the suction surfaces of gas turbine blades. Surface temperature measurements were conducted from the mid window in the test section, namely the corresponding viewing angel was $0^{\circ}$.

Figure 4 shows two series of the contour plots of the temperature drop from the initial wall to the cooled wall at different coolant injection rates, $1.0 \mathrm{~g} / \mathrm{s}, 1.5 \mathrm{~g} / \mathrm{s}, 2.0 / \mathrm{s}$, and $2.5 \mathrm{~g} / \mathrm{s}$, respectively. The upper series is from film cooling, and the lower is from transpiration cooling. The pixels numbered from 140 to 230 in the horizontal direction and from 65 to 145 in the vertical direction were processed and presented in these contour plots. The axis of film holes in the figure stood on the pixel number 217.

As shown in the lower plots, there was a significant variation of transpiration cooling effect on the surface of the porous tube. At the lowest coolant injection rate $1.0 \mathrm{~g} / \mathrm{s}$, the highest cooling effect could be found only in the range from the pixel number 215 to 225 at the bottom of the plot. With an increase in the injection rate, this highest effect range was regularly extended. This phenomenon may be explained by the fact that the exits of the injected chamber stood in opposition to this region, and the coolant with a higher momentum impinged on the bottom of the chamber, thus the cooling effect at the bottom of the porous tube was better than that at the top. In the horizontal direction
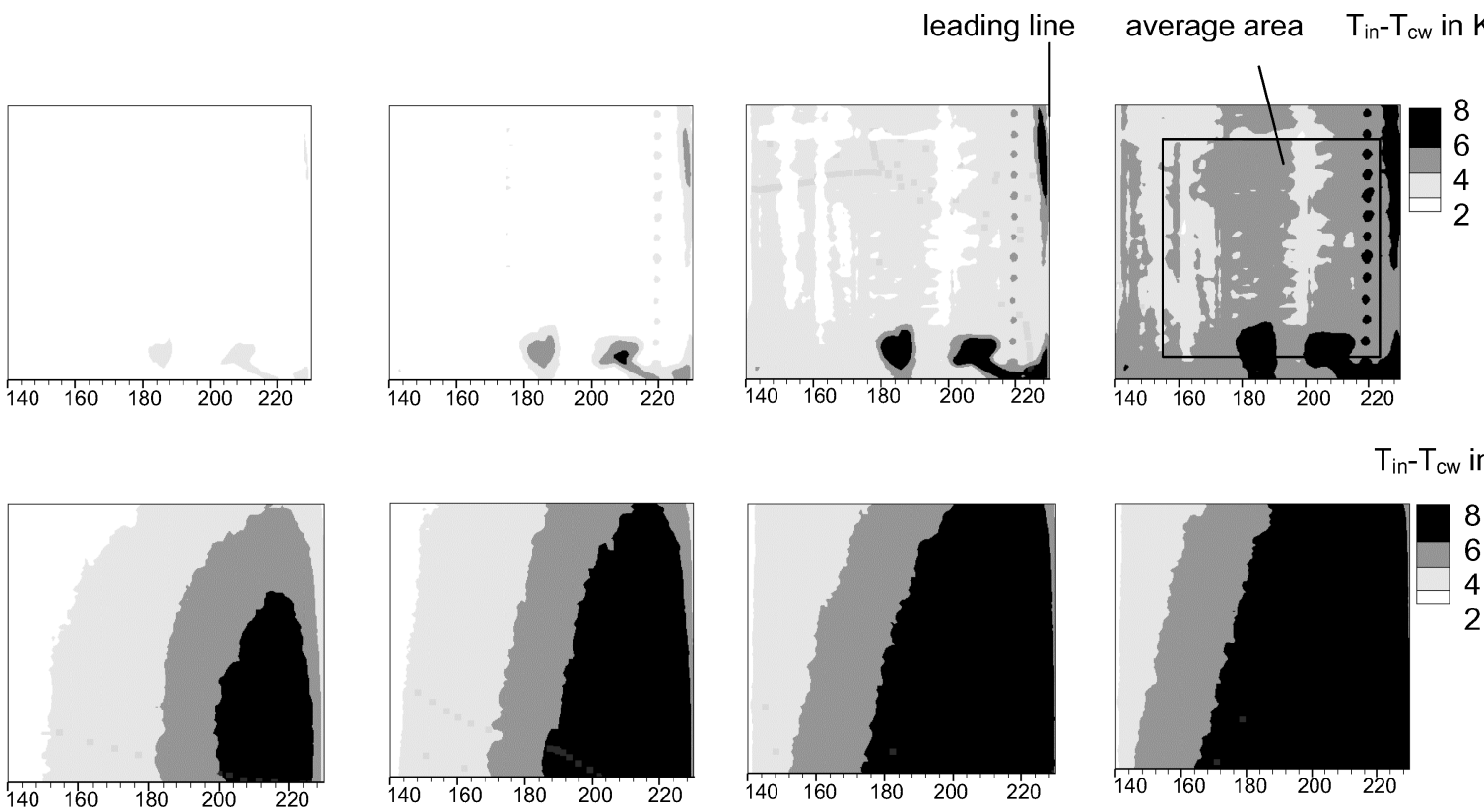

FIGURE 4

Local cooling effects distribution on the side of two tubes; above: film cooling. 
TABLE 2

Averaging Effect on Side of the Two Tubes

\begin{tabular}{lllll}
\hline & $1.0 \mathrm{~g} / \mathrm{s}$ & $1.5 \mathrm{~g} / \mathrm{s}$ & $2.0 \mathrm{~g} / \mathrm{s}$ & $2.5 \mathrm{~g} / \mathrm{s}$ \\
\hline FS & 0.0073 & 0.0112 & 0.0159 & 0.0228 \\
TS & 0.0245 & 0.0318 & 0.0401 & 0.0452 \\
\hline
\end{tabular}

of the plots, transpiration cooling effect was sensitive to the distance from the exits of the injected chamber. The cooling effect on the opposite side of the exits was the best, and further from the exits where the cooling effect was reduced.

In comparison with film cooling, the transpiration cooling effect was higher and the corresponding protected area was also larger at the same coolant injection flow rates. This result can also be demonstrated through a quantitative comparison of average effect in Table 2. The reason is that the effect of film cooling was only found on the outside surface covered by coolant film, the cooling effect was rapidly attenuated by the mixing of the coolant and the hot gas flow, whereas the effect of transpiration cooling was from the inner surface through the pores to the outside surface, the coolant had a low injection momentum, and slowly mixed with the hot gas flow, thus the cooling could maintain a higher effect.

\section{Cooling Effect in Trailing Region}

Figure 5 illustrates two series of the contour plots of the temperature drop from the initial wall to the cooled wall. Original IR images were captured from the downstream window of the specimens with a viewing angel of $-60^{\circ}$, while only the chambers at the trailing lines were supplied with gaseous coolant. The upper series is from trailing coolant injection cooling through discrete film holes, and the lower from transpiration cooling. The temperature drops in the plots were acquired from the pixel number 75 to 155 in vertical direction, from 246 to 282 in horizontal direction from the original images, and the trailing line stood on the pixel number 269.

Transpiration cooling covered a larger area than film cooling, thus its average cooling effect was higher than that of film cooling, as shown in Table 3. However, near the outlets of the film holes, the trailing injection cooling effect could locally achieve a high cooling effect, and in the axial direction of the solid tube, the temperature drop distributions were more homogenous. In other words, from pixel 266 to 272 , trailing injection cooling got a more homogenous cooling distribution, but the transpiration cooling effect at the bottom of the porous tube was higher than at the top of the tube. This phenomenon may be explained by the fact that blockage and endwall boundary layer effect caused bound vortices of the tube surface in the
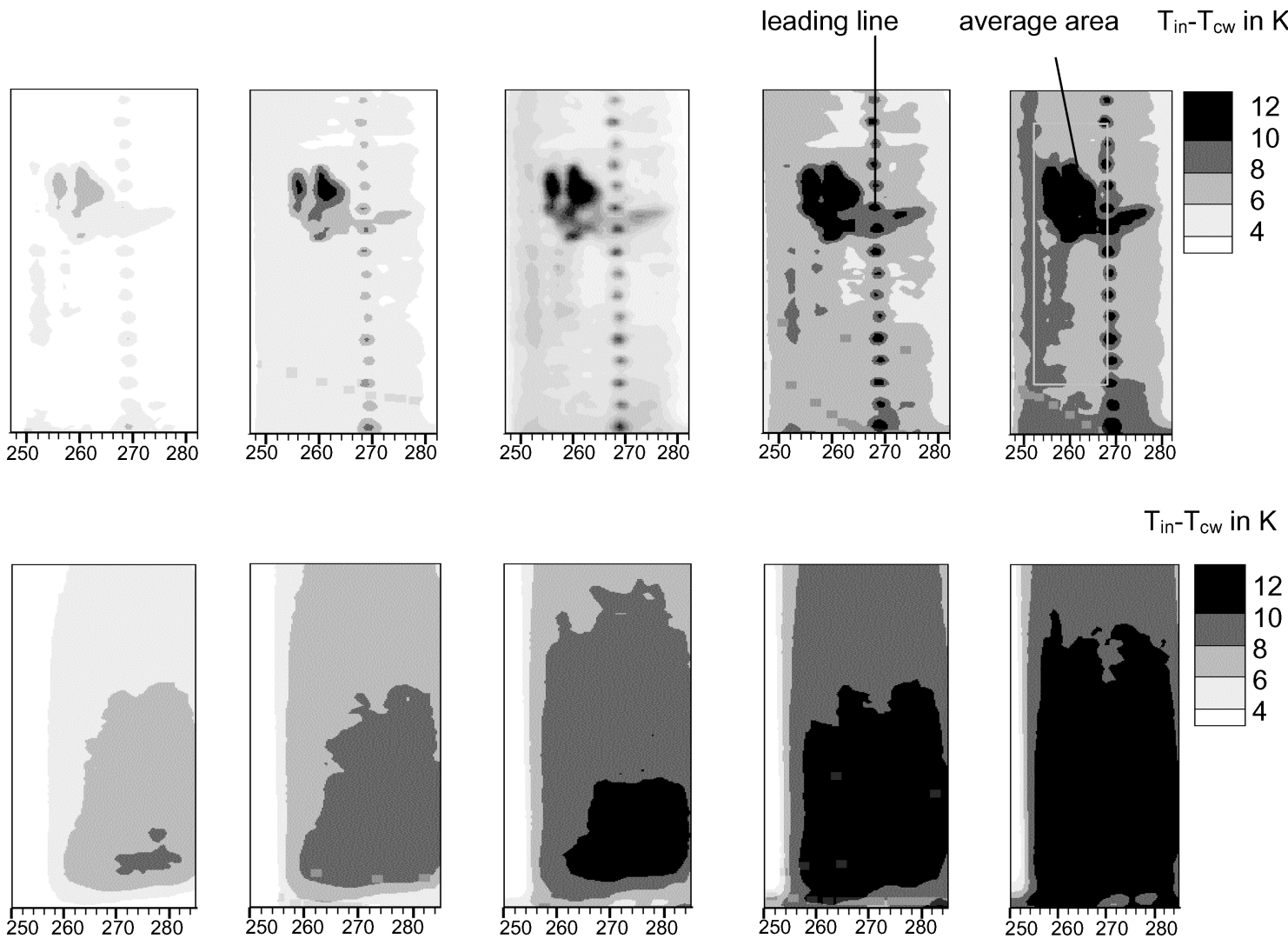
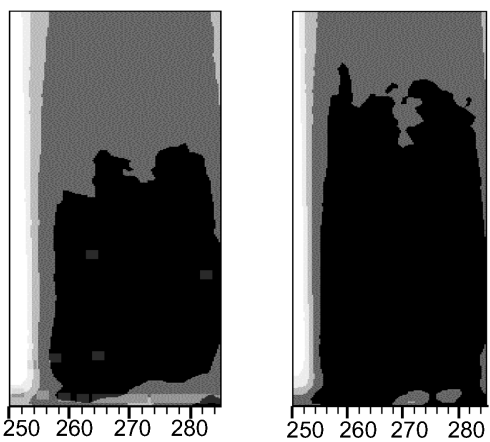

$\mathrm{T}_{\text {in }}-\mathrm{T}_{\mathrm{cw}}$ in $\mathrm{K}$

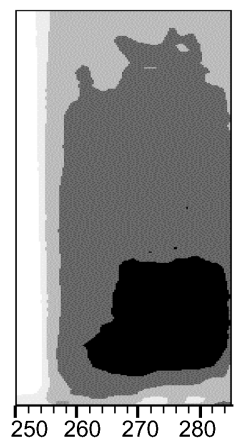

FIGURE 5

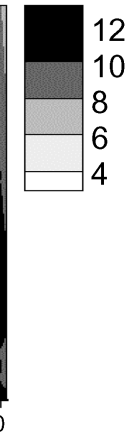

Local cooling effects in trailing stagnation region; above: film cooling. 
TABLE 3

Averaging Effect in Trailing Region

\begin{tabular}{llllll}
\hline & $1.0 \mathrm{~g} / \mathrm{s}$ & $1.5 \mathrm{~g} / \mathrm{s}$ & $2.0 \mathrm{~g} / \mathrm{s}$ & $2.5 \mathrm{~g} / \mathrm{s}$ & $3.0 \mathrm{~g} / \mathrm{s}$ \\
\hline FT & 0.0061 & 0.0101 & 0.0141 & 0.0177 & 0.0207 \\
TT & 0.0214 & 0.0312 & 0.0385 & 0.0463 & 0.0539 \\
\hline
\end{tabular}

trailing region, and the bound vortices have made pressure distribution not uniform in the axial direction, but the inner injection pressure of the coolant chamber was constant. According to Darcy's law, the mass flow of local effusion through a porous wall is directly proportional to the pressure drop through the wall, thus the level of coolant transpiration in the axial direction of the tube was not uniform. This phenomenon could also demonstrate that transpiration cooling effect was sensitive to the outside pressure distribution on the cooled wall, the location with higher pressure drop could obtain higher cooling effect.

In the case of trailing injection cooling of the solid tube, coolant with high velocity entered directly into the mainstream and weakened the bound vortices, thus trailing ejection cooling has obtained a better homogeneity in the axial direction. However, there was an island with a higher cooling effect at three-quarters span in the film cooling plots. This appearance was repeatedly tested and analyzed. An explanation is that there was a circumfluence with an opposite direction to coolant injection in this region due to the blockage effect, thus the injected coolant could not directly enter into the mainstream forming a small film cooling region.

\section{Comparison of Cooling Media}

In order to ensure water with a liquid state entered into the pores of the porous wall, the second experiment was conducted at a free stream temperature of $200^{\circ} \mathrm{C}$ with a mass flow rate of $400 \mathrm{~g} / \mathrm{s}$. In this experiment, pressurized air and pure water at temperatures $30^{\circ} \mathrm{C}$ and $20^{\circ} \mathrm{C}$ were successively injected into the same coolant chamber. Surface temperature was measured using the AGA 782 IR system. In order to observe the difference between the two different cooling media from the IR thermal images, the IR scanner was operated under the same optical conditions for the two measurements, and the original thermal images recorded with gray scale values were directly presented. A high gray scale value corresponds to a high temperature, and the calibration method was discussed in the first part of this article.

\section{Comparison on Porous Tube's Side}

The two chambers at $\pm 45^{\circ}$ from the leading line were supplied with gaseous coolant. For each chamber, coolant injection flow rates were $2.7 \mathrm{~g} / \mathrm{s}, 3.3 \mathrm{~g} / \mathrm{s}$, and $4 \mathrm{~g} / \mathrm{s}$, when the thermal images were captured, respectively. The water injection rates were adjusted until water evaporation cooling effects were close to the corresponding gaseous cooling effects, while the liquid injection flow rates were $0.08 \mathrm{~g} / \mathrm{s}, 0.16 \mathrm{~g} / \mathrm{s}$, and $0.32 \mathrm{~g} / \mathrm{s}$, respectively. That is, the ratios of gaseous and liquid injection rates were $33.75,20.625$, and 12.5 , respectively.

Figure 6 a shows the original images captured from $0^{\circ}$ viewing angel. The upper thermal images illustrate water evaporation cooling, and the lower gaseous transpiration cooling. In these images, the expression $\mathrm{L}, \mathrm{T}$, and $\mathrm{S}$ represent the positions of the leading line, trailing line, and sealings, respectively. From these images, it can be seen that the coverage of the two cooling media was almost identical, and the corresponding cooling effect was also very close.

The gray scale values were calibrated to obtain true surface temperatures, and local cooling effect distributions on the mid height of the porous tube were calculated, and illustrated in

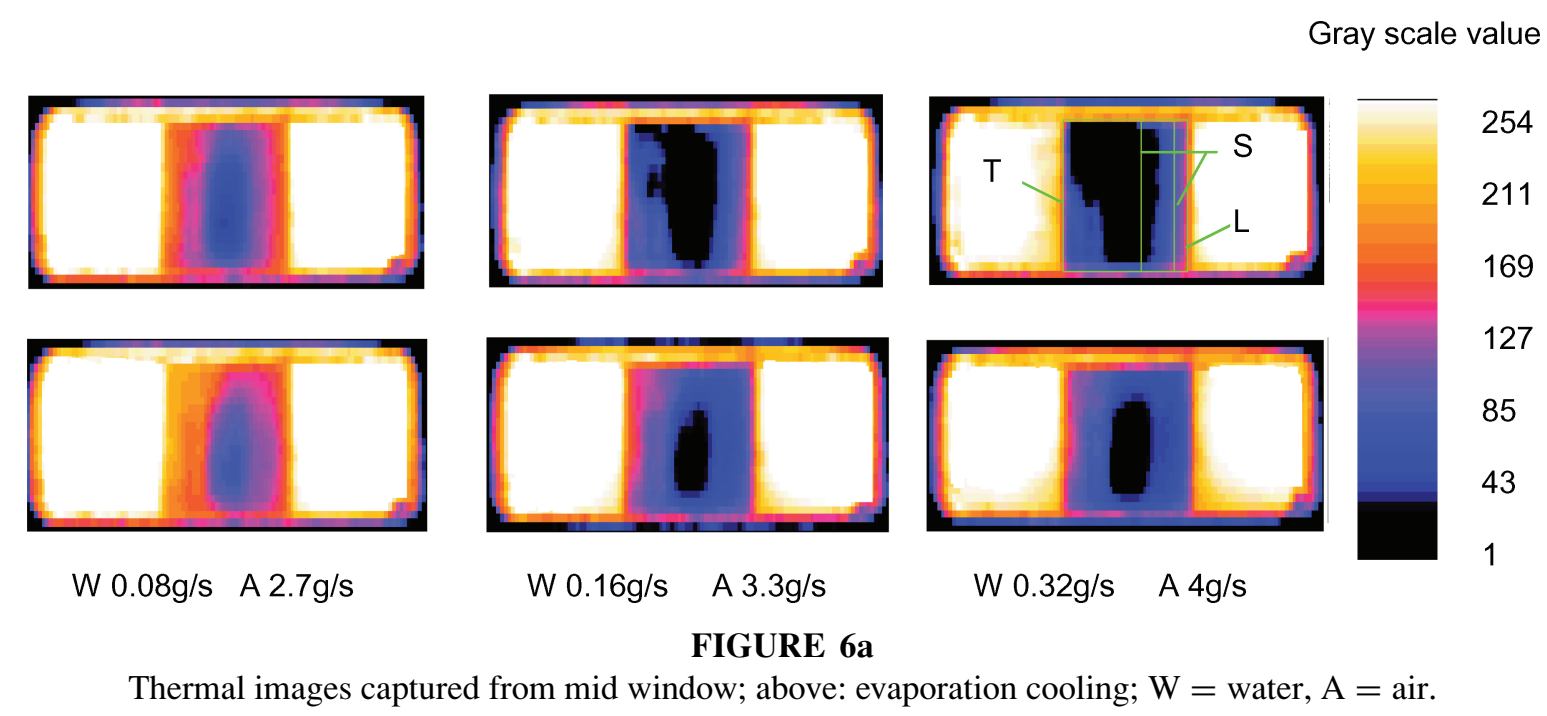




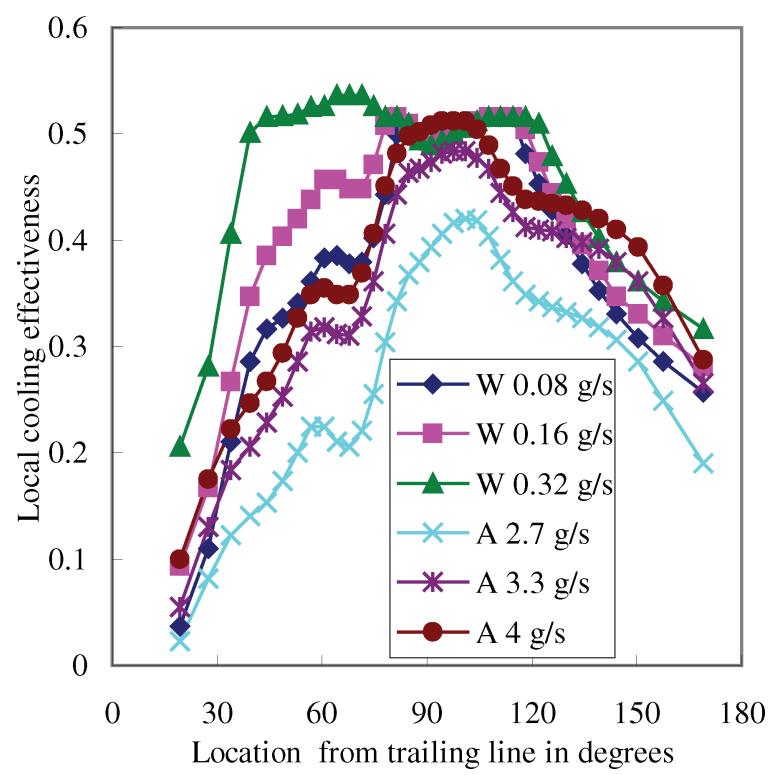

FIGURE 6b

Gaseous and liquid cooling effect on side.

Figure 6b. At the highest gaseous coolant injection flow rate of $4 \mathrm{~g} / \mathrm{s}$, gaseous cooling effect could locally achieve water evaporation cooling effect, and even exceed water evaporation cooling effect in the range from 120 to $160^{\circ}$.

\section{Comparison in Leading and Trailing Regions}

At the same injection flow rates of gaseous and liquid coolant, the same comparative experiments were carried out in the leading and trailing stagnation regions of the porous tube separately.

Figures $7 \mathrm{a}$ and $8 \mathrm{a}$ show the original IR thermal images captured from the upstream and downstream windows (with viewing angels of $\pm 60^{\circ}$ ), while the coolant chambers at the leading and

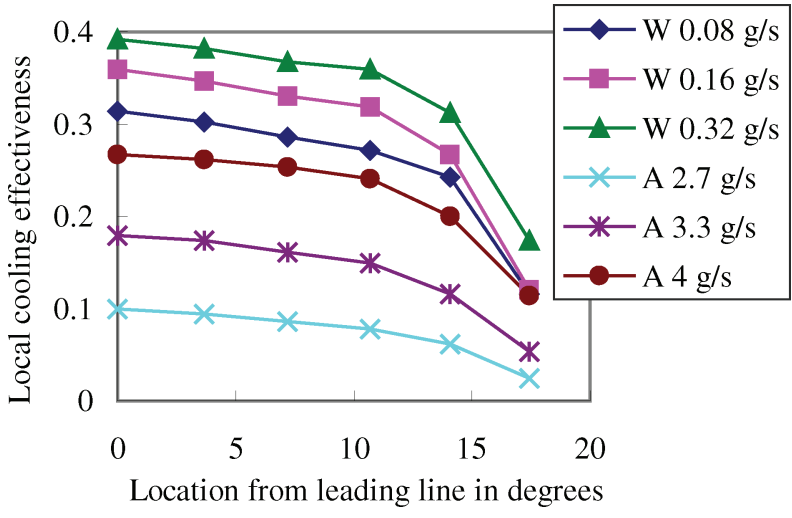

FIGURE 7b

Different cooling media in leading region.

trailing lines were supplied with different cooling media separately. The upper images illustrate liquid evaporation cooling effects, and the lower gaseous cooling. The coverage of liquid and gaseous cooling was almost identical, but the cooling effect of liquid evaporation was much better than that of gaseous transpiration. This result differs from that of the previous experiment conducted on the side of the porous tube, namely at the same ratios of gaseous and liquid injection rates, gaseous cooling effect in the two stagnation regions could not achieve the corresponding water evaporation effect on the side. Figures $7 \mathrm{~b}$ and $8 \mathrm{~b}$ quantitatively compared the difference between the two cooling media. Water evaporation cooling provided much higher effect than gaseous transpiration cooling. With the highest gaseous injection rate of $4 \mathrm{~g} / \mathrm{s}$, the corresponding effect was lower than the water evaporation cooling effect with the lowest water injection rate of $0.08 \mathrm{~g} / \mathrm{s}$.

Gaseous transpiration cooling effect in the two stagnation regions could not achieve the corresponding evaporation effect

Gray scale value

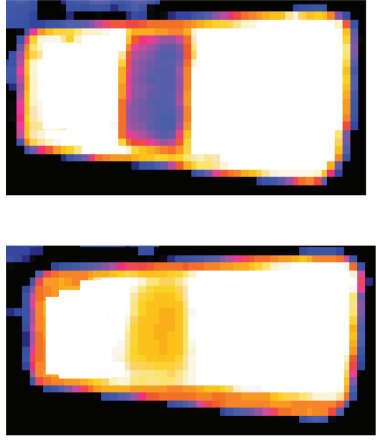

W $0.08 \mathrm{~g} / \mathrm{s} \quad$ A $2.7 \mathrm{~g} / \mathrm{s}$
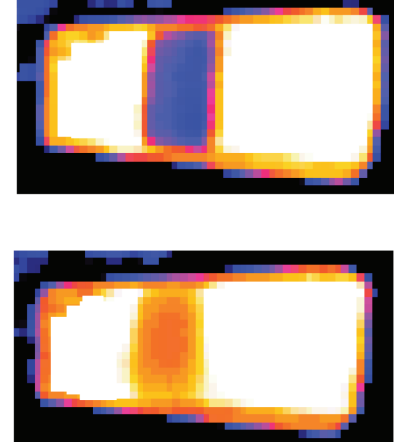

W $0.16 \mathrm{~g} / \mathrm{s} \quad$ A $3.3 \mathrm{~g} / \mathrm{s}$
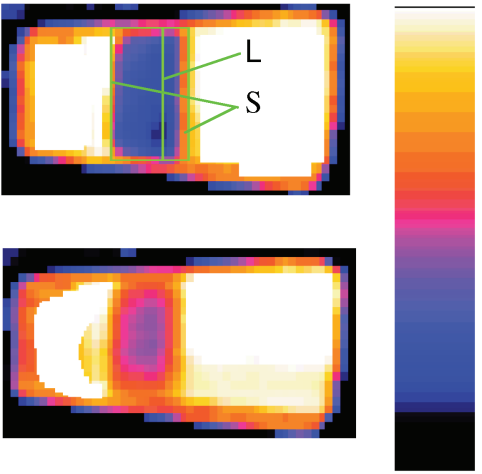

254

211

169

127

85

43

1

FIGURE 7a

Thermal images captured from upstream window; above: water evaporation cooling. 
Gray scale value

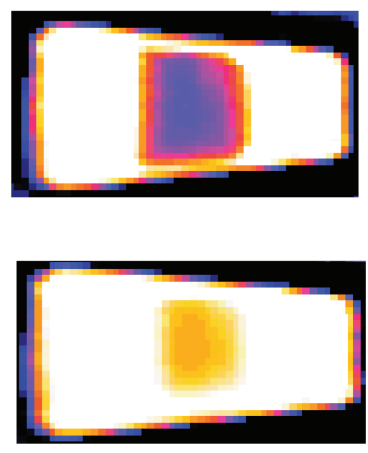

W $0.08 \mathrm{~g} / \mathrm{s} \quad$ A $2.7 \mathrm{~g} / \mathrm{s}$
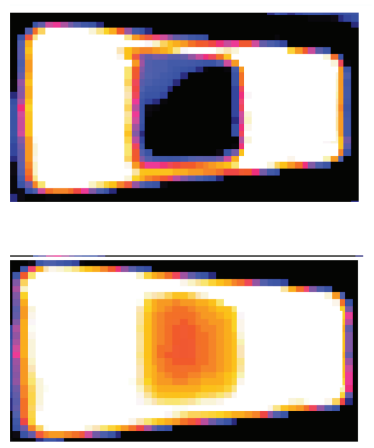

W $0.16 \mathrm{~g} / \mathrm{s}$

\section{A $3.3 \mathrm{~g} / \mathrm{s}$}
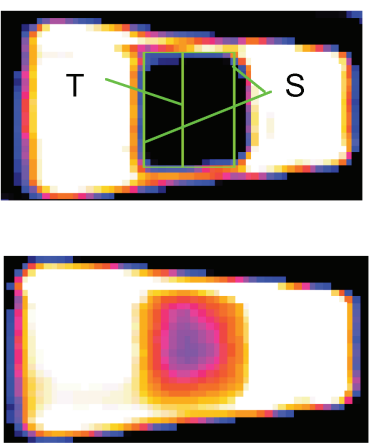

W $0.32 \mathrm{~g} / \mathrm{s} \quad$ A $4 \mathrm{~g} / \mathrm{s}$

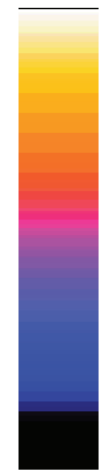

254

211

169

127

85

43

1

FIGURE 8a

Thermal images captured from downstream window; above: water evaporation cooling.

on the side of the porous tube at the same experimental conditions. The reason is that gaseous transpiration cooling effect depends on the flow nature of the boundary layer of the outside surface. In the stagnation regions, this flow nature was very weak, thus gaseous transpiration cooling effect in these regions was much lower than that on the side of the porous tube.

Water evaporation process could provide a very large heat sink in the two stagnation regions due to phase change. When the molecules on the liquid surface were hit by the hot gas flow, the energy of the molecules rose to a point that molecules could not maintain a liquid state resulting the phase change. This collision between gaseous and liquid molecules was independent upon the flow nature of the boundary layer, thus in the two stagnation regions liquid evaporation cooling could obtain high cooling effect. This discovery may be very usable in the future because it provides a possibility to solve the cooling problems in the stagnation regions of turbine blades.

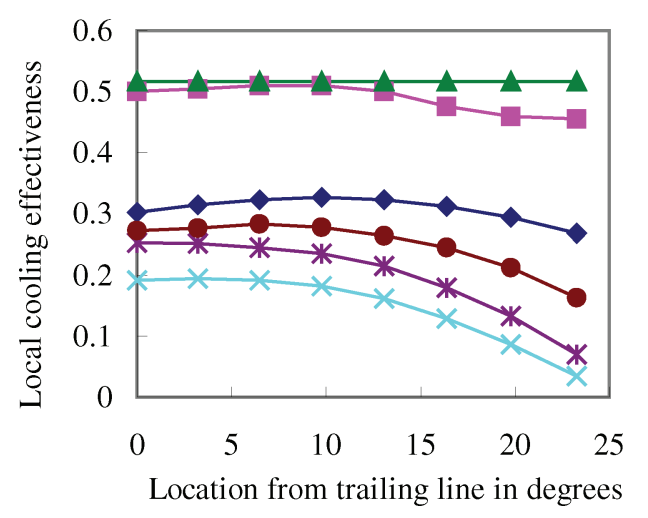

FIGURE 8b

Different cooling media in trailing region.

\section{CONCLUSIONS}

1. In comparison with film cooling, porous transpiration cooling using a gaseous cooling medium can provide a higher average cooling effect on the leading stagnation region, and on the side of the specimen. But in the trailing stagnation region, traditional injection cooling through discrete film holes may be better, especially for the turbine blades with thin trailing edges.

2. Porous transpiration cooling effectiveness is highly sensitive to the pressure distribution on the outside surface of the cooled wall and the exit position of coolant chamber. Thus, choosing a suitable position and shape of the coolant chamber in order to generate an optimal distribution of the cooling effect should be the ultimate aim of the cooling design.

3. Liquid coolant can be considered in substitution for gaseous cooling medium in the protection of the nozzle guide vanes of industrial gas turbine engines. The cooled areas of gaseous and liquid cooling media are almost identical, but liquid evaporation cooling effectiveness is much higher than gaseous cooling, especially in leading and trailing stagnation regions. This important feature of porous evaporation cooling makes it possible to eliminate the problems of stagnation regions in the current cooling techniques of gas turbine blades.

\section{REFERENCES}

Biesiadny, T. J., Klann, G. A., Clark, D. A., and Berger, B. 1987. Contingency power for small turboshaft engines using water injection into turbine cooling air, AIAA Paper No. 87-1906.

Leontiev, A. I. 1999. Heat and mass transfer problems for film cooling. ASME Journal of Heat Transfer 121:509-527.

Nirmalan, N. V., Weaver, J. A., and Hylton, L. D. 1998. An experimental study of turbine vane heat transfer with water-air cooling. ASME Journal of Turbomachinery 20:50-60.

Van Fossen, G. J. 1983. The feasibility of water injection into the turbine coolant to permit gas turbine contingency power for helicopter application. ASME Journal of Engineering of Power 105:635-642. 


\section{APPENDIX}

The relationship between the pixel number and the true orientation of the tube is shown in Figure 9. Where i, s, and $\mathrm{N}$ are a random pixel number, the ordinal number of borderline, and the total number on the imaged surface. The orientation angels $\theta$ corresponding to the pixel numbers were calculated through the following triangular equation:

$$
\theta_{\mathrm{i}}=\arccos \left(\cos \theta_{1} \frac{s-i}{s-1}\right) .
$$

The original thermal images in Figure 4 were captured from viewing angle $0^{\circ}$, the pixel number from 140 to 230 corresponds to the orientation angle $0^{\circ}$ to $180^{\circ}$ from the trailing line of the tube, and the film holes stand on the pixel number of 217. The original thermal images in Figure 3 were captured from viewing angle $60^{\circ}$, the leading line stands on the pixel number of 163 .

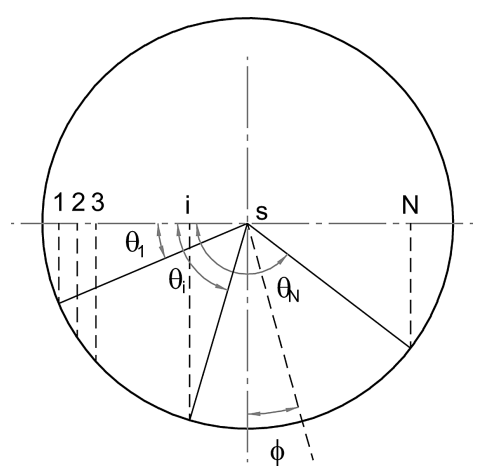

FIGURE 9

Relationship of pixel number and orientation.

The original thermal images in Figure 5 were captured from viewing angle $-60^{\circ}$, the trailing line on the pixel number of 269. Table 4 shows the true orientation angle.

TABLE 4

Relation of Orientation Angle and Pixel Number; $\mathrm{S}$ from $\phi=0^{\circ}, \mathrm{L}$ from $\phi=60^{\circ}, \mathrm{T}$ from $\phi=-60^{\circ}$, in Degree

\begin{tabular}{|c|c|c|c|c|c|c|c|c|}
\hline No. S & Angle & No. S & Angle & No. S & Angle & No. $T$ & No. L & Angle \\
\hline 140 & 0 & 177 & 79.76 & 214 & 130.12 & 282 & 150 & 56.68 \\
\hline 141 & 12.1 & 178 & 81.05 & 215 & 131.81 & 281 & 151 & 48.6 \\
\hline 142 & 17.15 & 79 & 82.34 & 216 & 133.54 & 280 & 152 & 42.39 \\
\hline 143 & 21.04 & 180 & 83.62 & 217 & 135.33 & 279 & 153 & 37.1 \\
\hline 144 & 24.34 & 181 & 84.9 & 218 & 137.67 & 278 & 154 & 32.38 \\
\hline 145 & 27.27 & 182 & 86.18 & 219 & 139.07 & 277 & 155 & 28.06 \\
\hline 146 & 29.93 & 183 & 87.45 & 220 & 141.06 & 276 & 156 & 24.58 \\
\hline 147 & 32.39 & 184 & 88.73 & 221 & 143.13 & 275 & 157 & 20.21 \\
\hline 148 & 34.69 & 185 & 90 & 222 & 145.31 & 274 & 158 & 16.57 \\
\hline 149 & 36.87 & 186 & 91.27 & 223 & 147.61 & 273 & 159 & 13.07 \\
\hline 150 & 38.94 & 187 & 92.55 & 224 & 150.07 & 272 & 160 & 9.68 \\
\hline 151 & 40.93 & 188 & 93.82 & 225 & 152.73 & 271 & 161 & 6.39 \\
\hline 152 & 42.83 & 189 & 95.1 & 226 & 155.66 & 270 & 162 & 3.16 \\
\hline 153 & 44.68 & 190 & 96.38 & 227 & 158.96 & 269 & 163 & 0 \\
\hline 154 & 46.46 & 191 & 97.66 & 228 & 162.85 & 268 & 164 & 3.12 \\
\hline 155 & 48.19 & 192 & 98.95 & 229 & 167.9 & 267 & 165 & 6.2 \\
\hline 156 & 49.88 & 193 & 100.24 & 230 & 180 & 266 & 166 & 9.25 \\
\hline 157 & 51.52 & 194 & 101.53 & & & 265 & 167 & 12.29 \\
\hline 158 & 53.13 & 195 & 102.84 & & & 264 & 168 & 15.32 \\
\hline 159 & 54.71 & 196 & 104.15 & & & 263 & 169 & 18.35 \\
\hline 160 & 56.25 & 197 & 105.47 & & & 262 & 170 & 21.39 \\
\hline 161 & 57.77 & 198 & 106.79 & & & 261 & 171 & 24.44 \\
\hline 162 & 59.26 & 199 & 108.13 & & & 260 & 172 & 27.52 \\
\hline 163 & 60.73 & 200 & 109.47 & & & 259 & 173 & 30.64 \\
\hline 164 & 62.18 & 201 & 110.83 & & & 258 & 174 & 33.8 \\
\hline 165 & 63.61 & 202 & 112.2 & & & 257 & 175 & 37.02 \\
\hline 166 & 65.03 & 203 & 113.57 & & & 256 & 176 & 40.32 \\
\hline 167 & 66.42 & 204 & 114.98 & & & 255 & 177 & 43.71 \\
\hline 168 & 67.8 & 205 & 116.39 & & & 254 & 178 & 47.21 \\
\hline 169 & 69.17 & 206 & 117.82 & & & 253 & 179 & 50.85 \\
\hline 170 & 70.53 & 207 & 119.27 & & & 252 & 180 & 54.67 \\
\hline 171 & 71.87 & 208 & 120.74 & & & 251 & 181 & 58.7 \\
\hline 172 & 73.21 & 209 & 122.23 & & & 250 & 182 & 63.02 \\
\hline 173 & 74.53 & 210 & 123.75 & & & 249 & 183 & 67.74 \\
\hline 174 & 75.85 & 211 & 125.29 & & & 248 & 184 & 73.03 \\
\hline 175 & 77.16 & 212 & 126.87 & & & 247 & 185 & 79.24 \\
\hline 176 & 78.46 & 213 & 128.48 & & & 246 & 186 & 87.32 \\
\hline
\end{tabular}



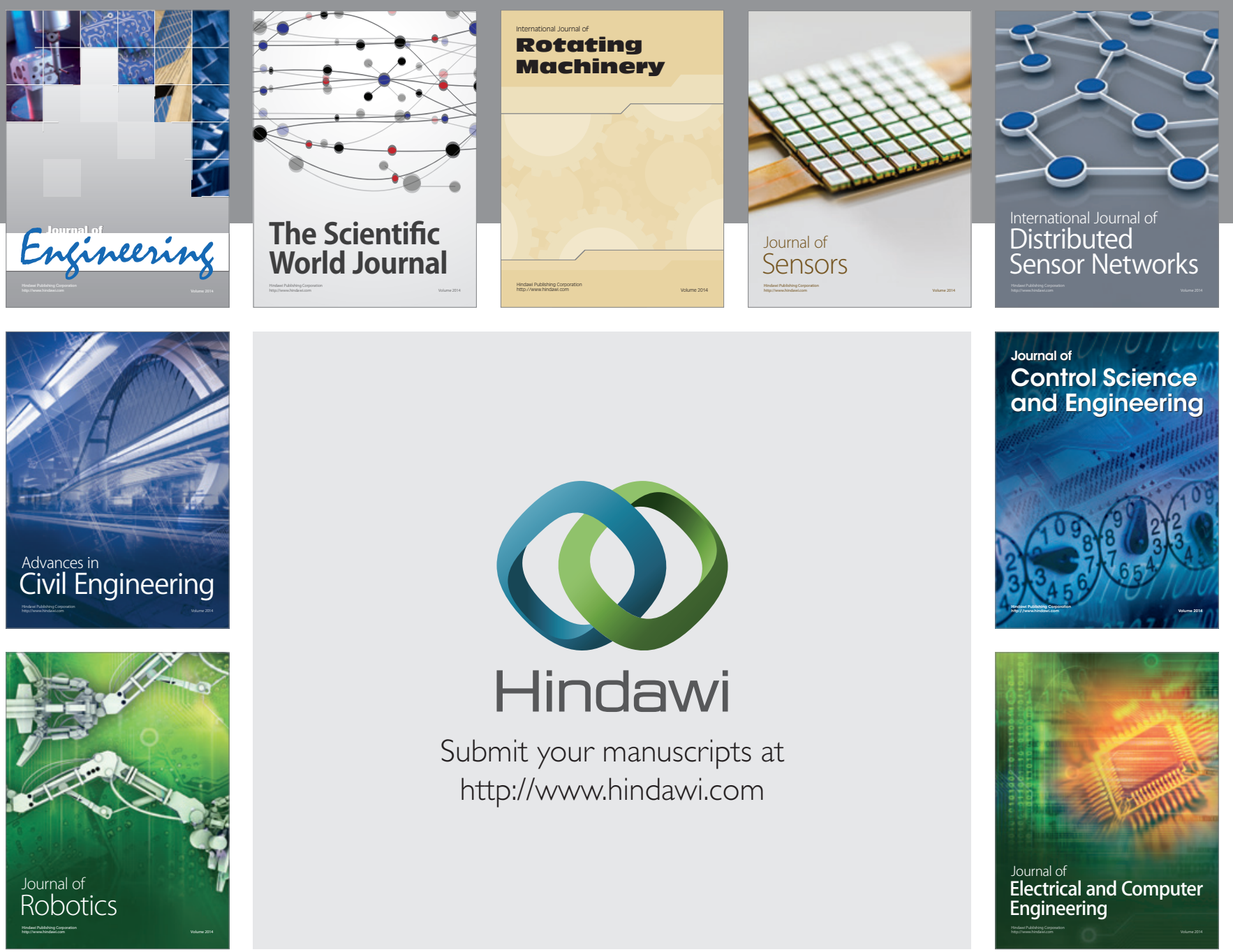

Submit your manuscripts at

http://www.hindawi.com
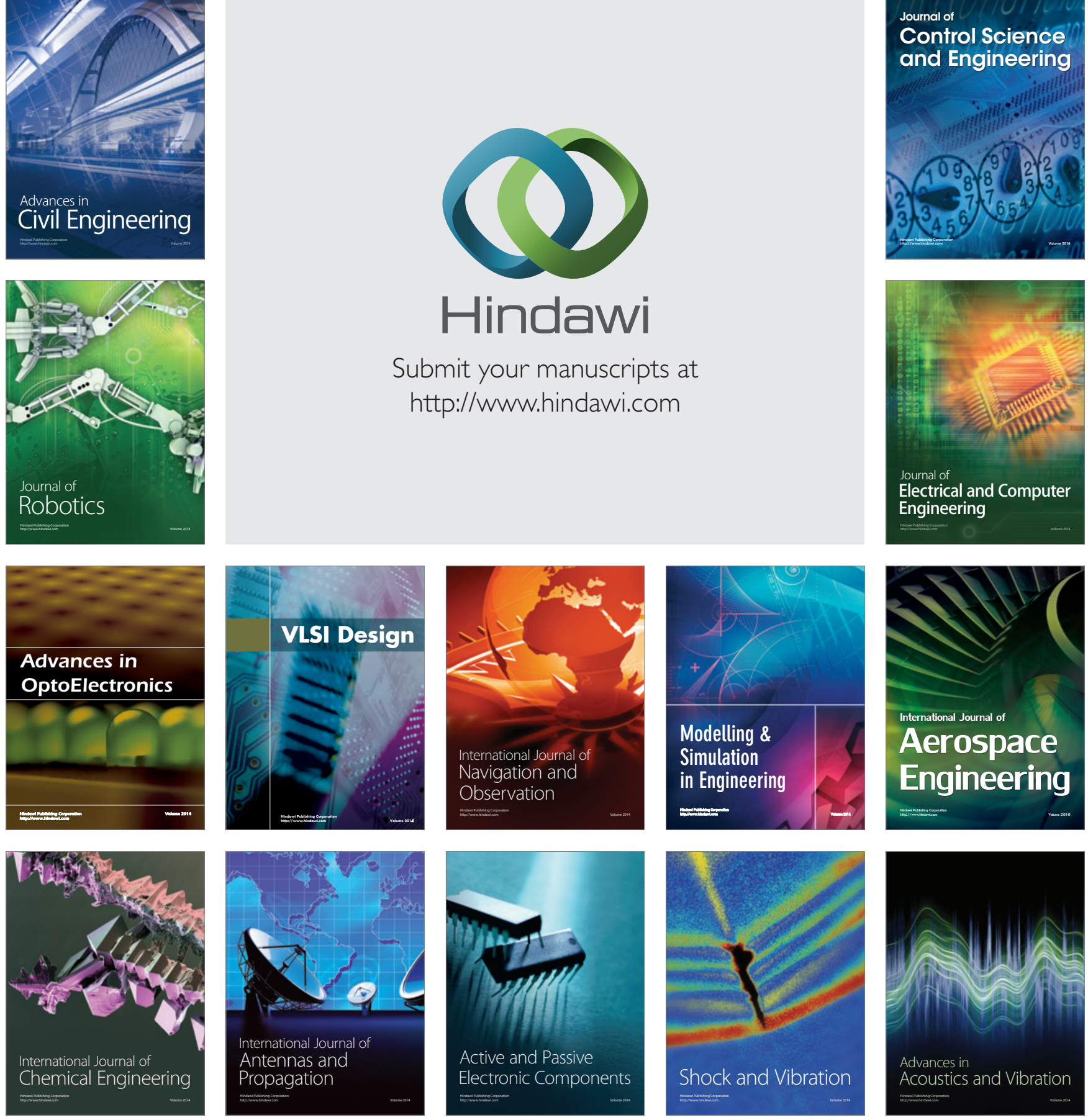